\title{
RADIOIODINE TREATMENT OF HYPERTHYROIDISM IN THE ELDERLY
}

\section{P1030}

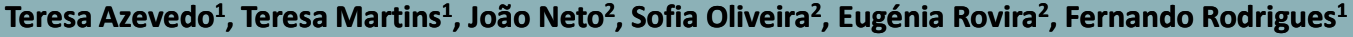

${ }^{1}$ Department of Endocrinology, ${ }^{2}$ Department of Nuclear Medicine. Portuguese Institute of Oncology (IPO), Coimbra, Portugal.

\section{BACKGROUND AND AIMS}

Treatment of hyperthyroidism in older adults is crucial to reduce its morbidity and mortality.

Radioactive iodine has been widely used in this age group due to its efficacy, safety and cost-effectiveness.

The aim of this study was to determine the efficacy of ${ }^{131}$ I for treatment of hyperthyroidism in the elderly.

\section{METHODS}

Retrospective study of 86 hyperthyroid patients aged $\geq 65$ years who performed radioiodine therapy for benign thyroid disease.

We excluded 22 patients who did not have a minimal follow-up of 1 year.

We evaluated the following parameters: gender, age, thyroid disease, clinical and laboratory situation at 1,3 and 5 years after treatment.

Dose of ${ }^{131}$ lodine $=[$ specific activity $(\mu \mathrm{Ci} / \mathrm{g}) \times$ glandular volume $(\mathrm{g})] / \%{ }^{131} \mathrm{I}$ fixation at $24 \mathrm{~h}$

\section{RESULTS}

$n=64$

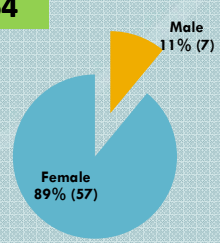

Figure 1. Sex distribution

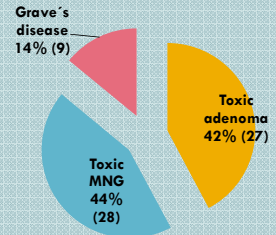

Figure 2. Thyroid disease

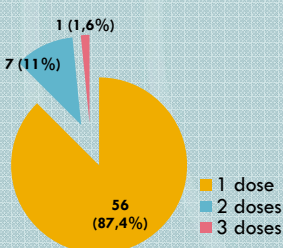

Figure 3. Number of ${ }^{131}$ doses

$\checkmark$ Mean age $( \pm S D)$ at the time of radioiodine treatment was $74.4 \pm 6.4$ years (range $65-89$ years)

$\checkmark$ One patient underwent surgery.

$\checkmark$ There were no significant complications due to radioiodine therapy.

Table 1. Comparison between calculated and administered ${ }^{131}$ doses. Paired samples Student's t-test.

\begin{tabular}{|c|c|c|c|c|}
\hline Follow-up & Situation & $\begin{array}{c}\text { Calculated } \\
\text { Activity MBq } \\
\text { (Mean } \pm \text { SD) }\end{array}$ & $\begin{array}{l}\text { Administered } \\
\text { Activity MBq } \\
\text { (MeantSD) }\end{array}$ & p \\
\hline \multirow{3}{*}{1 YEAR } & Euthyroidism & $463 \pm 408$ & $427 \pm 212$ & 0,336 \\
\hline & Hypothyroidism & $357 \pm 121$ & $372 \pm 96$ & 0,431 \\
\hline & Hyperthyroidism & $391 \pm 188$ & $373 \pm 146$ & 0,312 \\
\hline \multirow{3}{*}{3 YEARS } & Euthyroidism & $536 \pm 468$ & $460 \pm 221$ & 0,179 \\
\hline & Hypothyroidism & $333 \pm 149$ & $334 \pm 148$ & 0,746 \\
\hline & Hyperthyroidism & $435 \pm 232$ & $387 \pm 160$ & 0,362 \\
\hline \multirow{3}{*}{5 YEARS } & Euthyroidism & $574 \pm 435$ & $479 \pm 247$ & 0,236 \\
\hline & Hypothyroidism & $313 \pm 144$ & $317 \pm 141$ & 0,306 \\
\hline & Hyperthyroidism & $568 \pm 297$ & $449 \pm 133$ & 0,491 \\
\hline
\end{tabular}

Table 2. Comparison between the doses of iodine administered in $80 \%$ situations in which hyperthyroidism has been solved and situations in which it has not. Independent samples Student's t-test.

\begin{tabular}{|c|c|c|c|}
\hline Follow-up & $\begin{array}{l}\text { Hyperthyroidism } \\
\text { Adm Act MBq } \\
\text { (Mean } \pm S D)\end{array}$ & $\begin{array}{c}\text { Hypo or } \\
\text { Euthyroidism } \\
\text { Adm Act MBq } \\
\text { (Mean士SD) }\end{array}$ & $p$ \\
\hline 1 YEAR & $373 \pm 146$ & $421 \pm 202$ & 0,092 \\
\hline 3 YEARS & $387 \pm 160$ & $429 \pm 211$ & 0,614 \\
\hline 5 YEARS & $449 \pm 133$ & $418 \pm 225$ & 0,798 \\
\hline
\end{tabular}

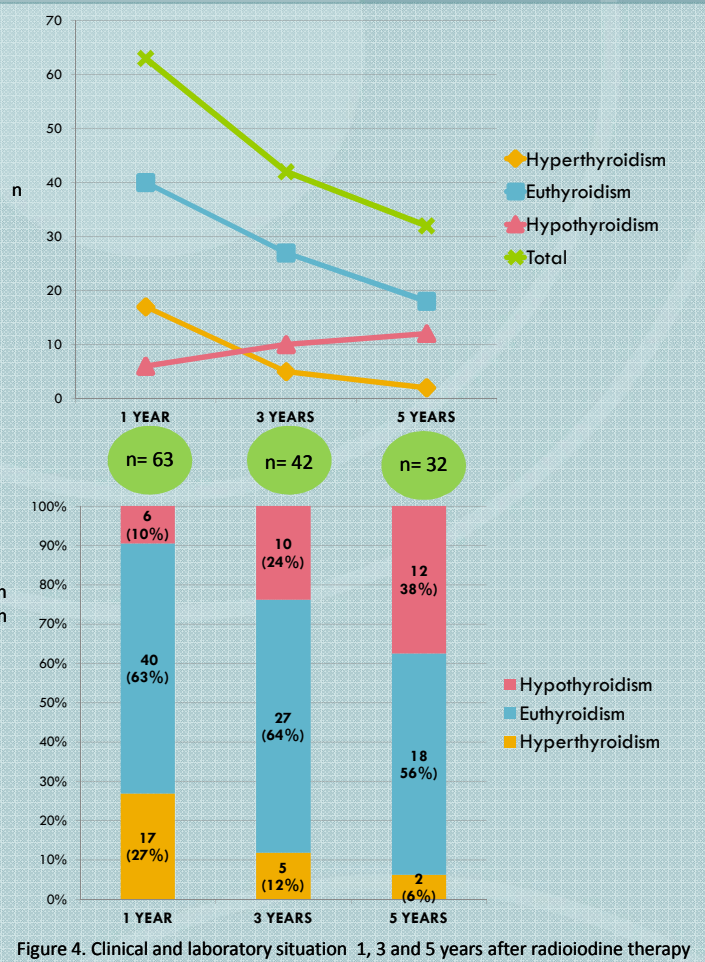

\section{DISCUSSION}

The majority of patients (87.6\%) needed only one dose of radioiodine.

During the follow-up there was:

- Increase of hypothyroid patietns: $10 \%, 24 \%$ and $38 \%$ at 1,3 and 5 years after radioiodine therapy, respectively;

- Reduction of hyperthyroid patients: $27 \%, 12 \%$ and $6 \%$ at 1, 3 and 5 years after radioiodine therapy, respectively;

- Maintenance of euthyroid patients: $63 \%, 45 \%$ and $56 \%$ at 1, 3 and 5 years after radioiodine therapy, respectively.

There were no statistically significant differences between administered doses of ${ }^{131} 1$ in cases of resolved hyperthyroidism versus the others.

\section{CONCLUSIONS}

Radioiodine therapy proved to be effective and safe to control hyperthyroidism in this age group.

The cell necrosis induced by radioiodine occurs gradually hence its effect may not be achieved immediately. 